\title{
Étude de la stabilité des collecteurs enterrés en maçonnerie
}

\section{О. THÉPOT}

Eau de Paris

2, av, de la Convention

94110 Arcueil

thepot@eaudeparis.fr
On examine, par des calculs simples, la stabilité d'un anneau en maçonnerie (collecteur circulaire) soumis à un champ de contraintes géostatique, sans prendre en compte l'interaction sol-structure, et on met ainsi en évidence un domaine de stabilité limité par deux valeurs du coefficient de pression horizontale des terres. L'étude est poursuivie avec la méthode des éléments finis (progiciel CESAR-LCPC), qui permet de prendre en compte l'interaction sol-structure. On met alors en évidence un nouveau domaine de comportement, appelé post-limite, situé à l'extérieur du domaine naturel de stabilité (appelé sub-limite), qui se caractérise par une forte plastification de l'anneau qui tend à se comporter comme un ensemble de 4 voussoirs articulés en forte interaction avec le sol encaissant. L'étude est reprise pour un collecteur en forme d'ovoïde, et on met aussi en évidence les mêmes domaines de comportement, mais avec des bornes différentes. On conclut que la stabilité d'un collecteur en maçonnerie (circulaire ou non circulaire) est directement liée à la raideur du sol encaissant et qu'il n'y a généralement pas d'équilibre possible sans prise en compte de l'interaction sol structure.

Mots-clés : collecteur enterré, maçonnerie, stabilité, interaction sol structure.

\section{Stability of masonry sewers}

one thus highlights a fieid of stability limited by two values of the horizontal earth pressure coefficient. The study is continued with the finite element method (software package CESARLCPC), which makes it possible to take into account the soilstructure interaction. One then highlights a new field of behavior, called post-limit, located outside the natural field of stability (called sub-limit), which is characterized by a strong plasticization of the ring, which tends to behave like a whole of 4 voussoirs articulated in strong interaction with the surrounding soil. The study is resumed for a egg shaped sewer, and one also highlights the same fields of behavior, but with different boundaries. It is concluded that the stability of a masonry sewer (circular or not circular) is directly related to the stiffness of the surrounding soil, and that there is generally no possible balance without taking into account of the soil-structure interaction.

Key words : sewer, masonry, stability, soil-structure interaction. 


\section{Introduction}

Les collecteurs en maçonnerie sont très nombreux et forment encore la majorité des ouvrages enterrés en milieu urbain. Ces ouvrages présentent une grande diversité de formes et de matériaux (Fig. 1); beaucoup datent du XIXe siècle et sont toujours en service. Les ouvrages les plus anciens comportent généralement une voûte plein cintre, des piédroits verticaux et un radier faiblement incurvé. L'épaisseur de ces ouvrages est souvent très élevée: $40 \mathrm{~cm}$ pour une ouverture de $1 \mathrm{~m}$, par exemple. Au milieu du XIX ${ }^{e}$ siècle est apparue la forme ovoïde (Hervieux, 1897), particulièrement bien adaptée au régime hydraulique des égouts qui transportent aussi bien des effluents avec un faible débit, que des pluies d'orage, qui peuvent saturer leur capacité hydraulique. L'étroitesse de la cunette assure une vitesse minimum aux effluents de temps sec et évite ainsi la formation de dépôts. La forme circulaire est mieux adaptée aux ouvrages qui fonctionnent en charge (car le rayon hydraulique est minimum) ou en régime permanent comme les aqueducs. Les dimensions internes varient typiquement de $0,80 \mathrm{~m}$ à $4 \mathrm{~m}$ pour l'ouverture et de $1,80 \mathrm{~m}$ à $4 \mathrm{~m}$ pour la flèche, la couverture de sol est souvent faible de l'ordre de $1 \mathrm{~m}$ à $2 \mathrm{~m}$.

Situés dans des sous-sols urbains de plus en plus encombrés, les collecteurs sont soumis aux sollicitations agressives du trafic qui a largement augmenté depuis le début du $\mathrm{XX}^{\mathrm{e}}$ siècle, et ils doivent subir les changements de leurs environnements proches: constructions d'ouvrages ou rechargement de la chaussée par exemple. Même si la majorité des ouvrages continuent à "fonctionner», leur état général se dégrade inexorablement, et comme la reconstruction n'est pas toujours possible pour des raisons de coût et d'impact social, la réhabilitation est dans de nombreux cas la solution adoptée par le maitre d'ouvrage.

Le problème de la réhabilitation et du renforcement, tel qu'il se pose vis-à-vis du dimensionnement, ne concerne que les ouvrages qui sont encore en équilibre stable en leur état actuel (pas d'effondrements; pas d'évolution reconnue ou prévisible vers une instabilité à court terme), Cet état actuel, considéré comme base du projet de réhabilitation, est souvent mal connu, les marges de sécurité qu'il contient sont incertaines (mais cependant supérieures à 1). Il peut exister des désordres dans l'ouvrage et dans le sol, et des modifications des pressions de contact sol/ouvrage qui seraient inacceptables dans un projet de construction neuve, mais qui ne sont pas incompatibles avec l'existence d'un état d'équilibre stable (RERAU, 2004). La difficulté principale est alors de quantifier l'état d'équilibre de l'ouvrage existant (ouvrage + sol) en vue de sa contribution à la résistance globale de l'ouvrage renforcé. La démarche habituelle consiste à rechercher l'état d'équilibre le plus défavorable (éventuellement hors du domaine de sécurité réglementairement exigible) à partir des actions permanentes et variables (y compris accidentelles) antérieures et actuelles. Cependant il existe une grande incertitude sur les actions que peut exercer le sol sur un ouvrage enterré, aussi bien en intensité qu'en répartition et il est donc essentiel de faire varier les paramètres dans des fourchettes larges.

\section{2}

\section{Étude de la stabilité d'un anneau en maçonnerie par des calculs simples}

\section{1}

\section{Critère de résistance d'une structure en maçonnerie}

La maçonnerie est un agencement de pierres ou de briques dont les joints entre blocs sont remplis d'un matériau de liaison destiné à rendre l'ensemble cohérent. Du point de vue mécanique la maçonnerie est un matériau composite qui supporte bien les efforts de compression et mal les efforts de traction. Dans la pratique, on suppose que la maçonnerie n'a pas de résistance en traction et a une résistance en compression égale à $\mathrm{R}_{\mathrm{c}}$. Ce critère est appelé critère de traction-compression.

La structure type en maçonnerie est une voûte que l'on découpe «virtuellement » en voussoirs séparés par des joints. Les joints doivent vérifier le critère de frotte-

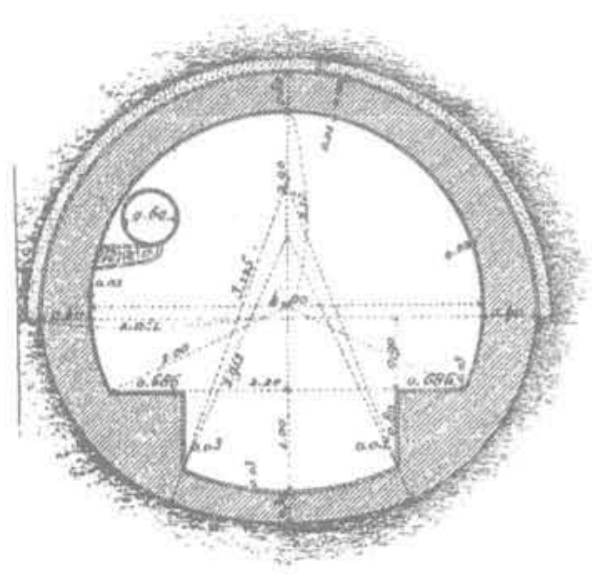

Fig. 269. - Collecteur de la Bièrre.

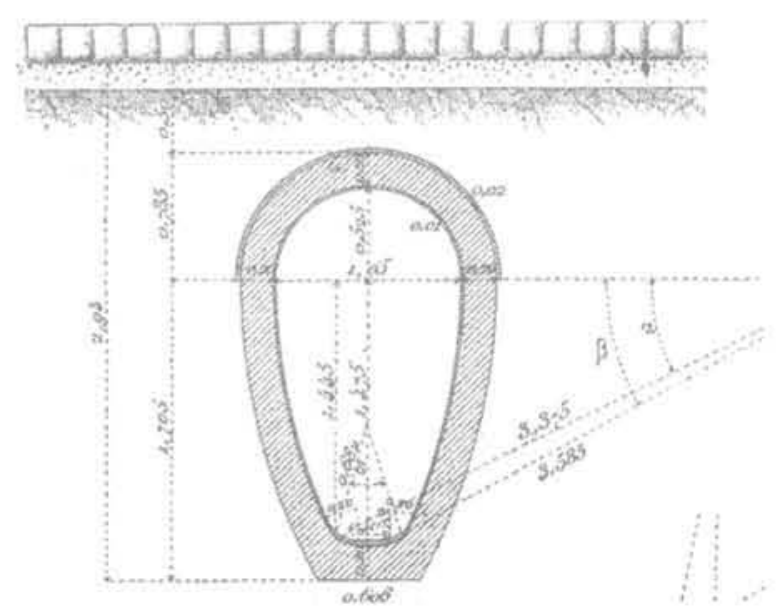

FIG, 1 Collecteurs d'assainissement (exemples). Sewer shapes examples. 
ment de Coulomb (avec une cohésion nulle). On montre (Delbecq, 1982) que le respect du critère de traction-compression implique que les efforts $\mathrm{M}$, N et T vérifient les deux inégalités suivantes sur chacun des joints :

$$
|M| \leq \frac{N \times h}{2}\left(1-\frac{N}{R_{c} h}\right) \quad|T| \leq N \tan \varphi
$$

où h est la hauteur du joint, $\varphi$ l'angle de frottement à l'interface et $\mathrm{R}_{\mathrm{c}}$ est la résistance en compression de la maçonnerie.

Le critère d'interface de Coulomb $T \leq N, \tan \varphi$ est généralement négligé, car il semble que l'on n'ait jamais observé de ruine par glissement des blocs les uns sur les autres.

Dans l'espace des sollicitations $\mathrm{M}, \mathrm{N}$ le domaine de résistance est un convexe limité par deux arcs de paraboles (Fig. 2a). Dans le cas d'une résistance infinie en compression (Fig. 2b), le domaine de résistance dégénère en un cône ouvert dans la direction de l'effort normal, dont les droites sont tangentes à l'origine aux arcs de paraboles (le domaine d'équilibre est donc plus grand). Les inégalités précédentes signifient simplement que la résultante des forces est à l'intérieur du joint et même suffisamment loin du bord si la résistance en compression est limitée.

\section{2}

\section{Modèle de calcul simplifié des actions dues au sol}

On suppose que les sollicitations (contraintes normales et de cisaillement) qui s'exercent sur l'extrados du collecteur sont en équilibre avec une distribution de contraintes dans le sol définie par des contraintes principales constantes orientées selon les axes horizontaux et verticaux. La contrainte verticale est notée $\mathrm{p}_{\mathrm{v}}$ et la contrainte horizontale $\mathrm{p}_{H}$ est égale à $\mathrm{k}$ fois la pression verticale (Fig. $3 \mathrm{a}$ ) et on néglige le gradient de pression verticale. Ce modèle simplifié de chargement est couramment utilisé dans les méthodes de calcul des conduites enterrées et des tunnels (AFTES, 1983).
Le coefficient de pression horizontale $k$ peut varier dans une large proportion, de 0 à 3 typiquement. Il n'est pas nécessairement égal au coefficient de pression horizontale des terres au repos noté habituellement $K_{0}$ car la distribution des contraintes dans l'environnement proche d'une conduite peut être fortement perturbée. Autrement dit, on ne peut pas relier k aux paramètres mécaniques du sol encaissant $(\varphi, C . .$.$) , c'est$ en fait un paramètre global qui caractérise le ratio entre la pression horizontale moyennée sur la hauteur de la conduite et la pression verticale. La pression normale p et la contrainte de cisaillement q à l'interface (Fig. 3b), obtenues par projection du champ de contraintes dans le sol sur l'extrados de l'anneau, sont données par les expressions suivantes :

$$
\mathrm{p}=\mathrm{p}_{\mathrm{v}}\left(\frac{1+\mathrm{k}}{2}-\frac{1-\mathrm{k}}{2} \cos 2 \theta\right) \quad \mathrm{q}=-\mathrm{p}_{\mathrm{v}} \frac{1-\mathrm{k}}{2} \sin 2 \theta
$$

\section{3}

\section{Équations de base}

Le rayon moyen du collecteur est noté $\mathrm{R}$ et son épaisseur h; on néglige les effets dus à la courbure. On considère deux sections potentiellement critiques: (C) en clé et (R) aux reins. La résistance des matériaux donne les valeurs suivantes des sollicitations agissantes (moment de flexion $\mathrm{M}$, positif s'il crée des tractions à l'intrados; effort normal N, positif s'il s'agit d'une compression; effort tranchant $V$ ):

-en clé:

$$
\begin{gathered}
M(C)=\frac{1-k}{4} p_{v} R^{2}\left(1-\frac{h^{2}}{4 R^{2}}\right) \\
N(C)=k p_{v}\left(R+\frac{h}{2}\right) \quad T(C)=0
\end{gathered}
$$

- aux reins :

$$
\begin{aligned}
& M(R)=-\frac{1-k}{4} p_{V} R^{2}\left(1-\frac{h^{2}}{4 R^{2}}\right) \\
& N(R)=p_{V}\left(R+\frac{h}{2}\right) \quad T(R)=0
\end{aligned}
$$

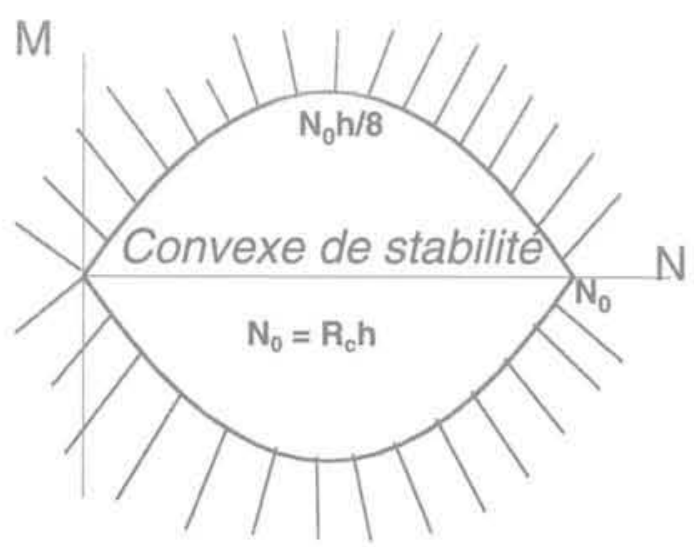

a) Résistance en compression finie

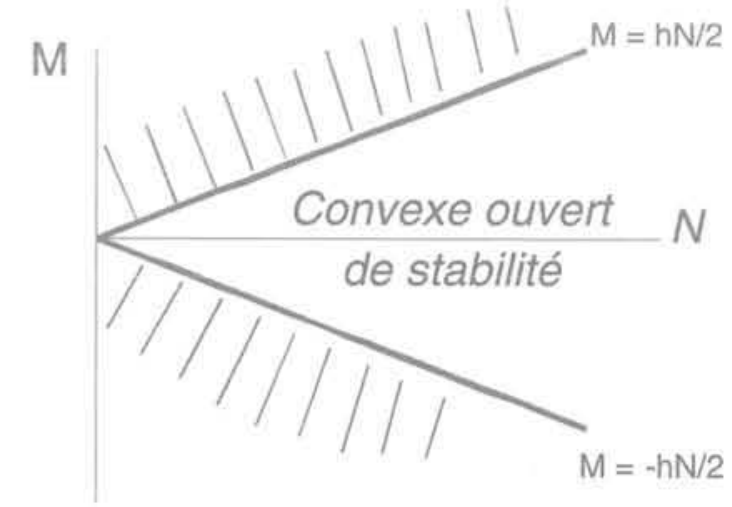

b) Résistance en compression infinie

EG. 2 Convexes de résistance d'une maçonnerie (résistance en traction nulle). Stability domain for the masonry material with zero tensile strenght. 


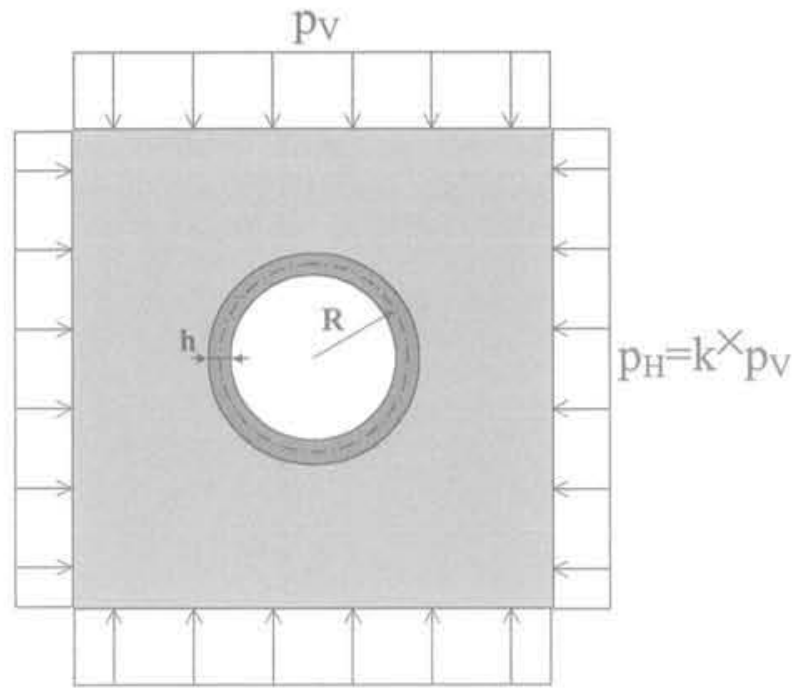

a) Contraintes initiales dans le sol

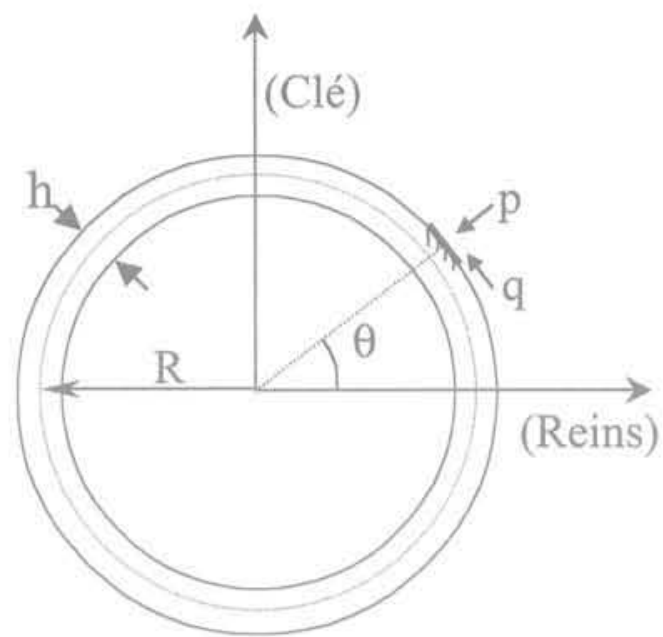

b) Contraintes à l'interface

FiG.3 Définition du chargement. Loading description.

- expression de l'excentricité e $=\mathrm{M} / \mathrm{N}$ en clé et aux reins :

$$
\begin{aligned}
& e(C)=\frac{1-k}{4 k} R\left(1-\frac{h}{2 R}\right) \\
& e(R)=-\frac{1-k}{4} R\left(1-\frac{h}{2 R}\right)
\end{aligned}
$$

\section{Compatibilité des équations d'équilibre avec le critère de résistance de la maçonnerie}

La compatibilité entre l'équilibre de la structure et le critère de résistance s'exprime facilement à partir de l'excentricité e (formules 5) et de l'inégalité (1) qui définit le convexe de stabilité de la maçonnerie. On exprime simultanément cètte compatibilité en clé et aux reins, qui sont les deux sections les plus sollicitées.

\subsection{1.}

\section{Cas d'une résistance à la compression infinie}

L'équilibre n'est possible que si: $-\frac{1}{2} \leq \frac{e}{h} \leq \frac{1}{2}$ ce qui donne 2 inégalités en clé et aux reins :

-en clé: $-\frac{1}{2} \leq \frac{1-k}{4 k} \frac{R}{h}\left(1-\frac{h}{2 R}\right) \leq \frac{1}{2}$

- aux reins : $-\frac{1}{2} \leq-\frac{1-k}{4} \frac{R}{h}\left(1-\frac{h}{2 R}\right) \leq \frac{1}{2}$

Les deux inégalités précédentes se traduisent par l'existence d'un coefficient de poussée minimum $\mathrm{k}_{\text {infr }}^{*}$ et un coefficient de poussée maximum $k_{\text {sup }}^{*}$ inverses l'un de l'autre et donnés par les expressions suivantes:

$\mathrm{k}_{\text {inf }}^{\infty}=\frac{1-\mathrm{h} / 2 \mathrm{R}}{1+3 \mathrm{~h} / 2 \mathrm{R}} \quad \mathrm{k}_{\text {sup }}^{\infty}=\frac{1}{\mathrm{k}_{\text {inf }}^{\infty}}$
Le coefficient de poussée minimum traduit la perte de l'équilibre en clé qui se produit avant la perte aux reins et inversement, le coefficient de poussée maximum traduit la perte d'équilibre aux reins qui se produit avant la perte en clé (Figs. $4 \mathrm{a}$ et $4 \mathrm{~b}$ ). Toutefois, lorsque l'équilibre limite est atteint dans l'une des sections critiques, et si le matériau présente un comportement plastique, le développement non symétrique de la fissuration provoque une redistribution "plastique» des moments, qui diminuent dans les sections les plus sollicitées et se reportent dans les sections les moins sollicitées. Bien entendu, ce mécanisme s'arrête dès que toutes les réserves d'équilibre sont consommées dans les quatre sections critiques, puisque l'on obtient alors un mécanisme (structure hypostatique) formé de quatre voussoirs articulés avec quatre rotules.

Si on appelle $\Delta \mathrm{M}$ la part de redistribution plastique du moment, on peut écrire :
$\left(M_{C}\right)_{D}=M_{C}+\Delta M$
en clé
$\left(M_{R}\right)_{p}=M_{R}+\Delta M$
aux reins

L'indice $\mathrm{p}$ indique les moments plastiques ou après redistribution plastique. On peut remarquer que $\Delta \mathrm{M}$ est négatif quel que soit $k$, car le moment de la section la plus critique est toujours positif.

Lorsque k est inférieur à 1, la section critique est en clé. La valeur maximum de $\Delta \mathrm{M}$ (en valeur absolue) est alors donnée par l'équation:

$$
\mathrm{M}_{\mathrm{C}}+\Delta \mathrm{M}=\mathrm{N}_{\mathrm{C}} \frac{\mathrm{h}}{2} \quad \begin{aligned}
& \text { (pour une résistance } \\
& \text { en compression infinie) }
\end{aligned}
$$

soit $\Delta M=-\frac{1-k}{4} p_{v} R^{2}\left(1-\frac{h^{2}}{4 R^{2}}\right)+k p_{v}\left(R+\frac{h}{2}\right) \frac{h}{2}$

En reportant le résultat précédent dans l'expression du moment plastique aux reins il vient :

$$
\left(M_{\mathrm{H}}\right)_{\mathrm{p}}=-\frac{1-\mathrm{k}}{2} \mathrm{p}_{\mathrm{v}} \mathrm{R}^{2}\left(1-\frac{\mathrm{h}^{2}}{4 \mathrm{R}^{2}}\right)+\mathrm{kp} \mathrm{v}\left(\mathrm{R}+\frac{\mathrm{h}}{2}\right) \frac{\mathrm{h}}{2}
$$

La réserve d'équilibre est entièrement consommée lorsque :

$$
\left(\mathrm{M}_{\mathrm{R}}\right)_{\mathrm{p}}=\mathrm{N}_{\mathrm{R}} \frac{\mathrm{h}}{2}
$$




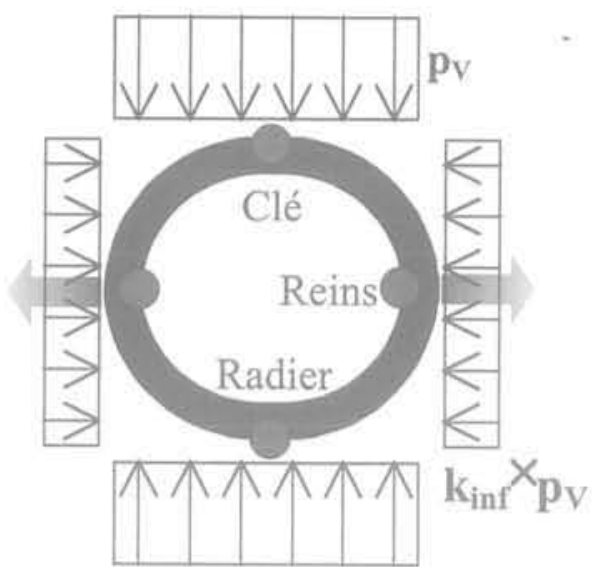

a) Divergence

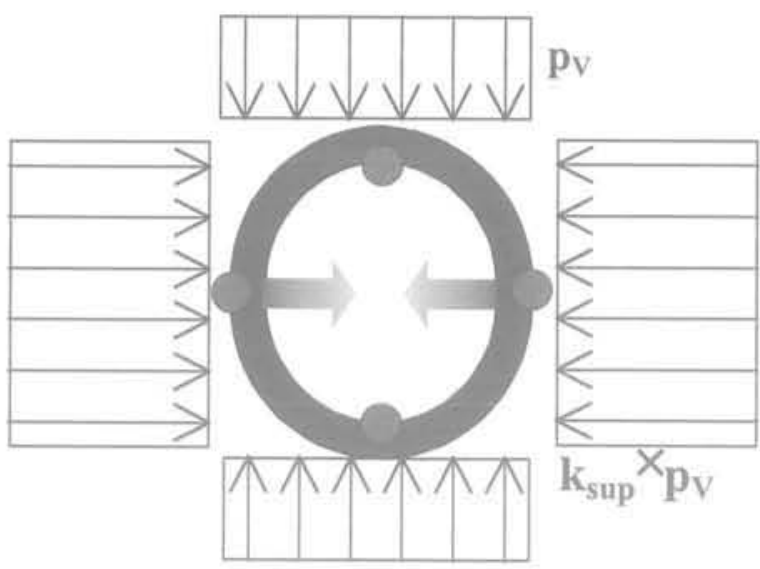

b) Convergence

FG.4 États limites d'équilibre d'un anneau en maçonnerie. Uitimate limit states of a masonry ring.

On tire de l'égalité précédente la valeur du coefficient de poussée minimum après redistribution:

$$
\left(k_{\text {inf }}^{*}\right)_{p}=\frac{1-3 h / 2 R}{1+h / 2 R}
$$

et celle du coefficient de poussée maximum:

$$
\left(k_{\text {sup }}^{\infty}\right)_{p}=\frac{1}{\left(k_{\text {inf }}^{*}\right)_{p}}=\frac{1+h / 2 R}{1-3 h / 2 R}
$$

On a évidemment $\left(\mathrm{k}_{\text {iff }}^{-}\right)_{\mathrm{p}}<\left(\mathrm{k}_{\text {sup }}^{-}\right)$

L'équilibre de l'anneau n'est possible que si le coefficient de poussée $k$ est compris entre les deux limites précédentes: $\left(\mathrm{k}_{\text {inf }}^{-}\right)_{\mathrm{p}}<\mathrm{k}<\left(\mathrm{k}_{\text {sup }}^{\prime \prime}\right)_{\mathrm{p}}$.

Dans l'espace $(\mathrm{h} / \mathrm{R}, \mathrm{k})$, le domaine d'équilibre de l'anneau en maçonnerie est borné par une ligne de poussée minimum et une ligne de poussée maximum (Fig. 5). On remarquera que la valeur $\mathrm{k}=1$ est dans le domaine d'équilibre (puisqu'elle correspond au confinement isotrope de l'anneau qui ne crée que des efforts normaux et pas de moments).

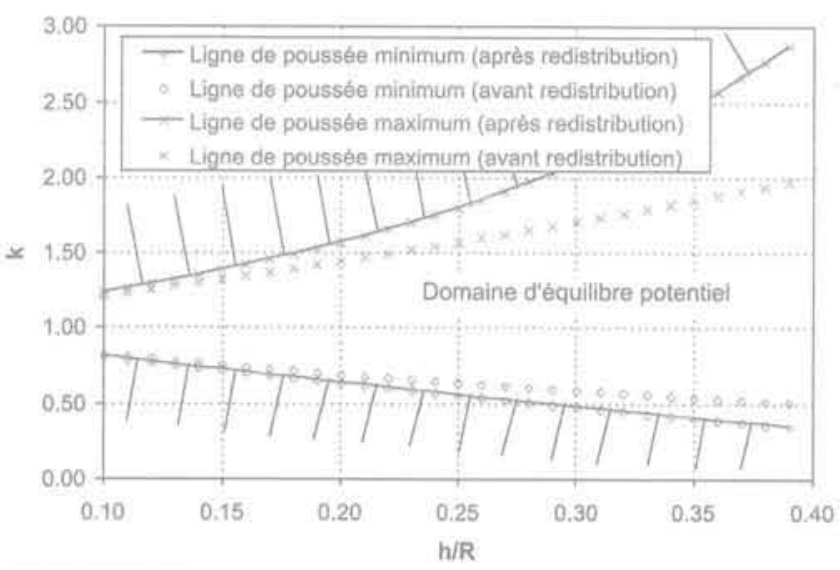

FG, 5 Domaine d'équilibre potentiel d'un anneau en fonction du ratio épaisseur sur rayon et du coefficient de pression horizontale du sol.

Potential field balance of a masonry ring versus thickness ratio and coefficient of horizontal earth pressure $\mathrm{k}$.

\section{8}

\section{Cas de la résistance en compression finie}

La compatibilité du critère de résistance tractioncompression avec l'équilibre des sections critiques donne les deux inégalités suivantes :

-en clé :

$$
-\frac{1}{2}\left(1-\frac{k p_{V} R}{h R_{c}}\right) \leq \frac{1-k}{4} \frac{R}{h}\left(1-\frac{h}{2 R}\right) \leq \frac{1}{2}\left(1-\frac{k p_{V} R}{h R_{c}}\right)
$$

- aux reins :

$$
-\frac{1}{2}\left(1-\frac{p_{V} R}{h R_{C}}\right) \leq-\frac{1-k}{4} \frac{R}{h}\left(1-\frac{h}{2 R}\right) \leq \frac{1}{2}\left(1-\frac{p_{V} R}{h R_{C}}\right)
$$

oủ $R_{c}$ est la résistance en compression de la maçonnerie.

Lorsque k est inférieur à 1, la section critique est en clé et la valeur minimale de k compatible avec l'équilibre de la section est donnée par l'équation suivante:

$$
\frac{1-k_{\text {inf }}}{4 k_{\text {inf }}} R\left(1-\frac{h}{2 R}\right)=\frac{h}{2}\left(1-\frac{k_{\text {inf }} p_{V} R}{h R_{C}}\right)
$$

Si on pose $\alpha=p_{v} / R_{c}$ on peut exprimer, sous la forme d'un développement limité, la valeur minimum de $k$ en fonction de celle calculée pour une résistance infinie :

$$
\mathrm{k}_{\text {inf }}^{\mathrm{R}_{\mathrm{i}}}=\mathrm{k}_{\text {inf }}^{-}+\frac{1}{2}\left(\mathrm{k}_{\text {inf }}^{-}\right)^{2}\left(1+3 \mathrm{k}_{\text {inf }}^{-}\right) \alpha+o[\alpha]^{2}
$$

Pour la grande majorité des collecteurs, la hauteur de couverture est comprise entre 1 et $10 \mathrm{~m}$, ce qui correspond à une pression verticale $p_{y}$ comprise entre 0,02 et $0,2 \mathrm{MPa}$, et la résistance en compression $R_{c}$ varie dans une fourchette comprise entre 3 à $10 \mathrm{MPa}$. Par conséquent, a varie dans une fourchette comprise entre 0 et 0,1 . Pour la valeur de 0,1 , la correction ne dépasse pas $5 \%$ et est négligeable dans la grande majorité des cas. On peut donc dire que la résistance en compression de la maçonnerie a peu d'influence sur les bornes du domaine d'équilibre, c'est à dire que l'équilibre d'un anneau se réduit à un problème purement géométrique.

Avec un jeu minimal d'hypothèses, on a donc montré qu'il existe un domaine d'équilibre borné par un 
coefficient de pression minimum $\mathrm{k}_{\text {int" }}$ et un coefficient de pression maximum $k_{\text {sup }}$ (Fig. 6) dont les expressions sont simples et ne dépendent que de l'épaisseur et du rayon de l'anneau (pour une résistance en compression infinie). Ces deux coefficients limites sont associés à un mécanisme de rupture à quatre rotules de type cléradier-reins (Figs, $4 a$ et 4 b). A l'extérieur de ce domaine, il n'y a pas d'équilibre possible quelle que soit l'intensité du chargement, à l'intérieur du domaine l'équilibre est toujours possible. Cependant, cette première analyse ne donne aucune information sur l'état de déformation (et de contrainte) de l'anneau, et ne permet pas de prendre en compte l'interaction sol-structure qui modifie, dans un sens généralement favorable, la distribution des pressions à l'interface sol-structure. On présente donc par la suite l'analyse élasto-plastique (avec la méthode des éléments finis) d'un anneau en interaction avec un massif de sol.

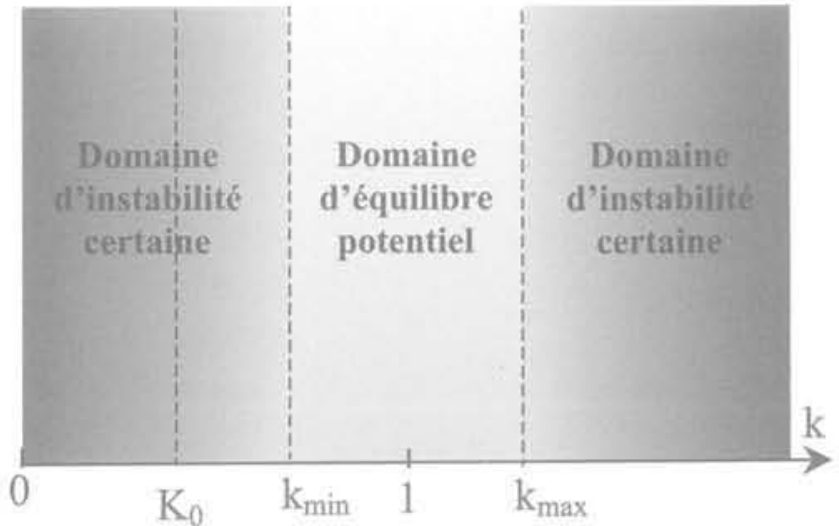

FG.6 Domaine d'équilibre potentiel et domaines d'instabilité d'un anneau en maçonnerie. Potential balance field and unsteady balance fieid of a masonry ring versus coefficient of horizontal pressure $\mathrm{k}$.

\section{Étude de la stabilité d'un anneau en maçonnerie en interaction avec un massif de sol par la méthode des éléments finis}

\section{Introduction}

On examine maintenant l'équilibre d'un anneau de maçonnerie en interaction avec un massif de sol homogène, isotrope et élastique, et soumis à un chargement identique à celui de l'étude précédente. On suppose que le massif de sol possède un état de contrainte initial caractérisé par une contrainte verticale $p_{v}$ et une contrainte horizontale $\mathrm{kxp}$ constantes (on néglige le gradient vertical de contrainte) et que l'anneau de maçonnerie est mis en place immédiatement après l'excavation (on ne tient pas compte du déconfinement du sol).

Contrairement aux recherches classiques de charges limites, on ne fait pas varier ici l'intensité du chargement qui reste fixe, mais un paramètre géomé- trique qui caractérise la forme du chargement (le coefficient $\mathrm{k}$ ),

\section{2}

\section{Caractéristiques du modèle aux éléments finis}

La modélisation par éléments finis a été réalisée avec le logiciel CESAR-LCPC (Humbert, 1989) et l'option «LAM » du module de résolution élasto-plastique "MCNL » (Mestat, 1993). Le matériau maçonnerie est entendu dans son acception la plus large, c'est-àdire un matériau dont la résistance en traction est très faible ou nulle, et dont la résistance en compression est élevée. On a donc choisi la loi du critère parabolique qui est classiquement utilisée pour modéliser le béton non armé. Le critère s'écrit en fonction des invariants du tenseur des contraintes:

$$
F\left(\sigma_{i j}\right)=J_{2}\left(\sigma_{i j}\right)-\frac{R_{c}-R_{i}}{3} L_{1}\left(\sigma_{i j}\right)-\frac{R_{c} R_{i}}{3}
$$

où $J_{2}\left(s_{i}\right)$ est le déviateur des contraintes, $I_{1}$ est la trace du tenseur des contraintes, $\mathrm{R}$ est la résistance en compression et $\mathrm{R}$, la résistance en traction.

Dans l'espace des contraintes principales, la surface de rupture est un paraboloïde ayant pour axe la droite d'équation : $\sigma_{1}=\sigma_{2}=\sigma_{3}$. La loi d'écoulement est associée, c'est-à-dire que le potentiel plastique est confondu avec le critère (élasto-plasticité parfaite). La partie élastique du comportement est définie par l'élasticité linéaire isotrope de Hooke. Au total, la loi comporte donc quatre paramètres: $E, n, R$ et $R$, Les calculs ont été réalisés en contraintes planes plutôt qu'en déformations planes pour une raison qui est liée au critère parabolique, mais qui n'a pas d'incidence sur les conclusions de l'étude. Le sol a été modélisé en élasticité linéaire, la figure 7 représente le maillage utilisé.

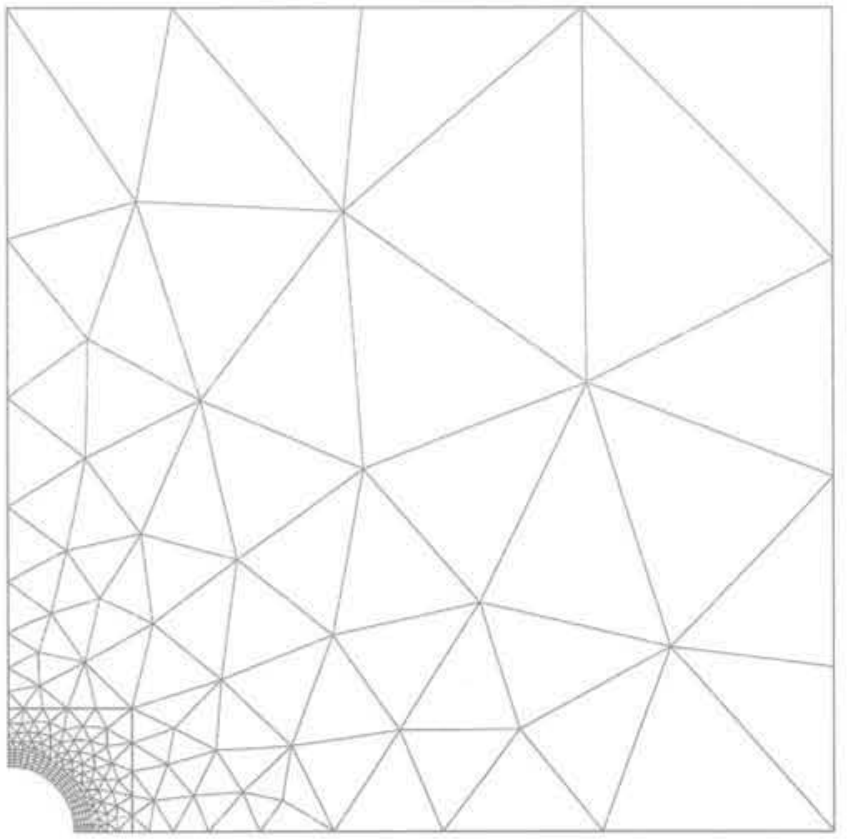

FIG. 7

Dessin du maillage d'éléments finis (logiciel CESAR-LCPC).

Drawing of the finite elements mesh ifinite element package CESAR-LCPC). 
Les caractéristiques mécaniques du massif de sol et les contraintes initiales sont les suivantes:

- élasticité linéaire en contrainte plane:

- module d'Young : 0,5 et $30 \mathrm{MPa}$;

- coefficient de Poisson : 0,33 ;

$\sigma_{y y}=-100 \mathrm{kPa} ; \sigma_{x x}=-k \times 100 \mathrm{kPa} ; \sigma_{x y}=0 \mathrm{KPa} ; \sigma_{z z}=0 \mathrm{kPa}$; $k$ varie entre 0 et 1 .

La fourchette de module 5-30 MPa est représentative de ce que l'on peut mesurer avec un pressiomètre dans la tranche 0-10 $\mathrm{m}$ en site urbain. La valeur 0 a été ajoutée pour faire une comparaison avec le calcul précédent.

Les caractéristiques géométriques de l'anneau de maçonnerie sont les mêmes que dans le problème précédent, le module de Young varie de 1000 à $10000 \mathrm{MPa}$, la résistance en traction est nulle et la résistance en compression est égale à $30 \mathrm{MPa}$, ce qui figure ici une résistance infinie, c'est à dire que l'on exclut de l'étude la ruine par écrasement plastique (mais on discute de cette éventualité plus loin).

Les valeurs des coefficients de poussée minimum et maximum (avec redistribution plastique) prédites par le calcul manuel sont:

$$
\begin{gathered}
\mathrm{k}_{\text {inf }}=\frac{1-3 \mathrm{~h} / 2 \mathrm{R}}{1+\mathrm{h} / 2 \mathrm{R}}=0,6 \\
\mathrm{k}_{\text {sup }}=\frac{1}{\mathrm{k}_{\text {min }}}=1,67
\end{gathered}
$$

La fourchette 1 000-10 000 MPa couvre une large gamme de qualité de maçonnerie. Dans le cadre de cette étude, un module de $1000 \mathrm{MPa}$ est une valeur caractéristique d'une maçonnerie médiocre avec des joints dégradés, un module de $3000 \mathrm{MPa}$ représente la maçonnerie moyenne la plus courante et le module de 10000 est celui d'une très bonne maçonnerie.

\section{3}

\section{Analyse des résultats en déplacements: domaines sub-limite et post-limite}

La figure 8 représente les variations de l'ovalisation horizontale (déplacement radial maximal mesuré au niveau des reins) de l'anneau en fonction de $k$ pour les trois modules de sol 0,5 et $30 \mathrm{MPa}$ et un module de $10000 \mathrm{MPa}$ pour la maçonnerie. On constate tout d'abord qu'en absence d'interaction (le module du sol est égal à zéro), la courbe diverge au voisinage de $k=0,6$ qui est la valeur limite inférieure prévue par l'approche manuelle. Ce résultat est conforme aux théorèmes de l'analyse limite (Salençon, 1983).

On constate ensuite que la raideur du sol (l'interaction sol-structure) permet à l'anneau de «passer» la valeur limite $\mathrm{k}_{\text {inf }}$ (égale à 0,6 ) qui provoquait la ruine plastique. Cependant, si l'anneau trouve une position d'équilibre pour toutes les valeurs de $\mathrm{k}$ comprises entre 0 et 1 , les déplacements et l'étendue des zones plastifiées augmentent considérablement quand $\mathrm{k}$ dépasse la valeur limite et tend vers zéro. On est donc amené naturellement à distinguer deux domaines de fonctionnement, selon que k est inférieur ou supérieur à la borne inférieure $k_{\text {irf }}$ : le domaine post-limite (ou domaine d'adaptation) pour les valeurs inférieures et le domaine sub-limite (ou domaine d'équilibre naturel) pour les valeurs supérieures.

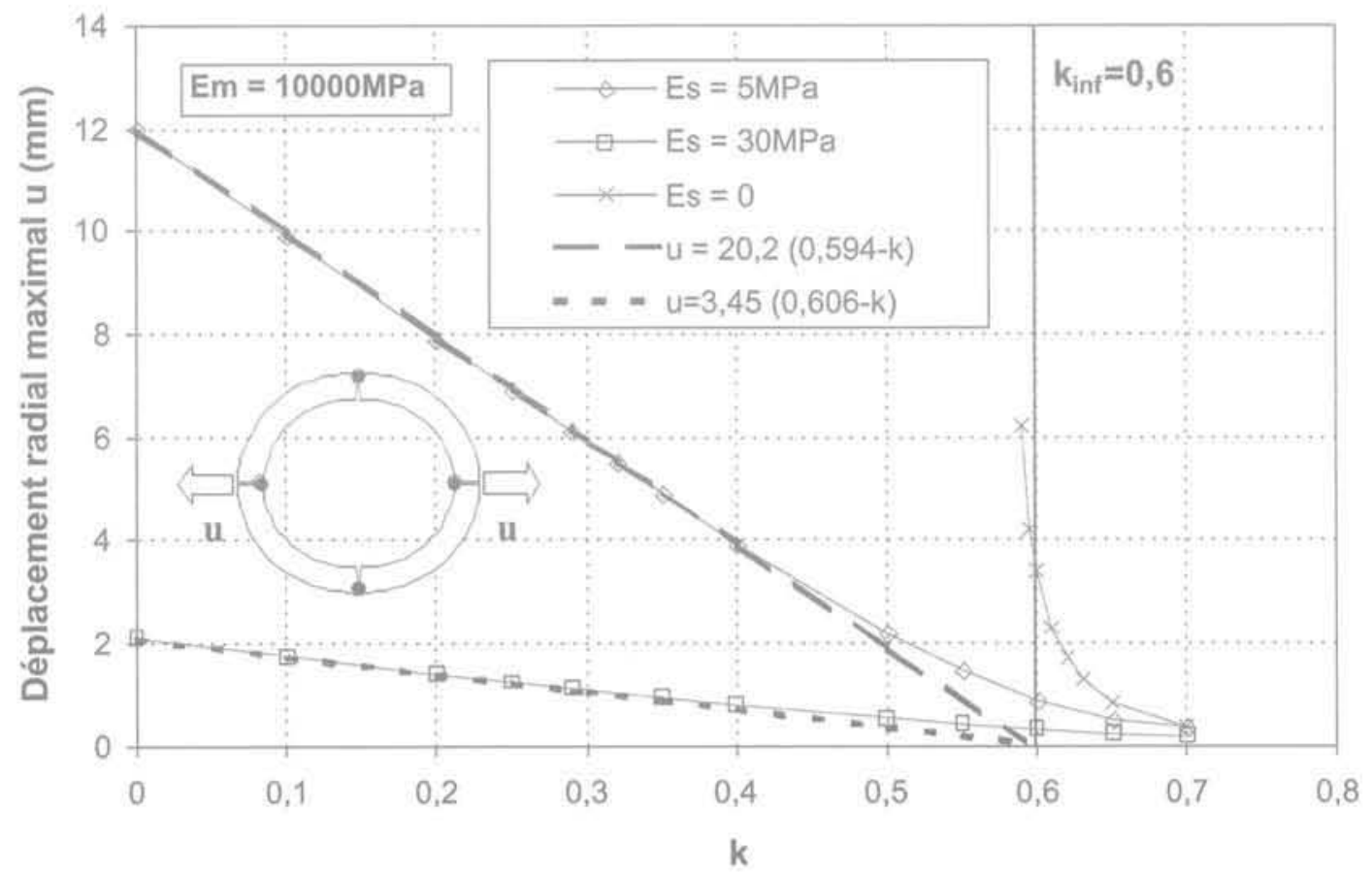

FIG. 8 Résultats des calculs par éléments finis : déplacement radial maximal de l'anneau en fonction du coefficient de pression horizontale $\mathrm{k}$.

Finite element results : maximal radial deflection of the ring at springlines versus coefficient of horizontal earth pressure $\mathrm{k}$. 


\section{3 .1}

\section{Analyse des résultats dans le domaine post-limite}

L'examen des résultats dans le domaine post-limite montre que les déplacements varient quasi linéairement avec k (Fig. 8). Ce résultat est a priori surprenant, car l'anneau est fortement plastifié, mais il se comprend très bien si on suppose que l'anneau est formé de 4 voussoirs articulés avec 4 rotules plastiques situées en clé-reins et radier comme le représente la figure 4. En effet, le système de voussoirs forme alors un mécanisme (système hypostatique) dont la déformation ne dépend que du module du sol et du coefficient $k$.

Si on suppose que la pression de réaction du sol est proportionnelle au déplacement radial de l'anneau et si on néglige les déplacements élastiques, on peut montrer que le déplacement radial maximum peut se mettre sous la forme suivante:

$$
\frac{\mathrm{u}}{\mathrm{R}} \approx \mathrm{A} \frac{\mathrm{P}_{\mathrm{v}}}{\mathrm{E}_{\mathrm{s}}}\left(\mathrm{k}_{\text {inf }}-\mathrm{k}\right) \text { pour } \mathrm{k}<\mathrm{k}_{\text {int }}
$$

où $E_{\text {s }}$ est le module du sol et $A$ est une constante. On a représenté sur la figure 8 , les droites ajustées sur les résultats CESAR avec la formule (22).$$
3.3 .2
$$

\section{Analyse des résultats dans le domaine sub-limite}

Dans le domaine sub-limite, l'anneau est faiblement plastifié et son comportement se rapproche de celui d'un anneau élastique. La modélisation bidimensionnelle d'un anneau élastique en interaction avec un massif de sol élastique est un problème classique pour lequel il existe de nombreuses solutions analytiques (Einstein et Schwartz, 1979). Si on néglige les déformations dues à l'effort normal, le déplacement radial maximum de l'anneau est donné par:

$$
\frac{\mathrm{u}}{\mathrm{R}}=\mathrm{p}_{\mathrm{v}} \frac{(1-\mathrm{k}) / 12 \mathrm{~K}_{\mathrm{b}}}{1+\alpha \mathrm{F}}
$$

où:

$\mathrm{K}_{b}$ est le module de rigidité en flexion de l'anneau ou rigidité annulaire;

$F$ est le coefficient de couplage en flexion;

$\alpha$ dépend de la nature de l'interface et du coefficient de Poisson du sol et est proche de 0,1 (pour une interface collée).

$$
\begin{aligned}
& K_{b}=\frac{E I}{\left(1-v_{c}^{2}\right) R^{3}} \\
& F=\frac{E_{s}}{K_{b}\left(1-v_{s}^{2}\right)}
\end{aligned}
$$

où :

El et $v$ sont le produit d'inertie et le coefficient de Poisson de la paroi:

$\mathrm{E}_{\mathrm{s}}$ et $\mathrm{n}_{\mathrm{s}}$ sont le module et le coefficient de Poisson du sol.

En schématisant, on peut dire que dans le domaine post-limite, l'anneau se comporte comme s'il était formé de quatre voussoirs articulés, dont l'ovalisation est inversement proportionnelle au module du sol. Dans le domaine sub-limite, l'anneau a un comportement plutôt monolithique qui se rapproche de la solution élastique. Le passage de la limite s'accompagne d'une forte plastification, qui est contenue par l'interaction avec le sol. La figure 9 schématise les deux domaines de comportement de l'anneau.

\section{4}

\section{Analyse des sollicitations}

La figure 10 représente les courbes de moment pour les sections clé-radier et les sections de reins. On constate que les courbes de moment en clé-radier présentent un maximum pour $\mathrm{k}$ proche de $\mathrm{k}_{\text {inf }}$ et qu'elles s'infléchissent ensuite vers une asymptote linéaire qui tend vers une valeur un peu supérieure à 0 , Les

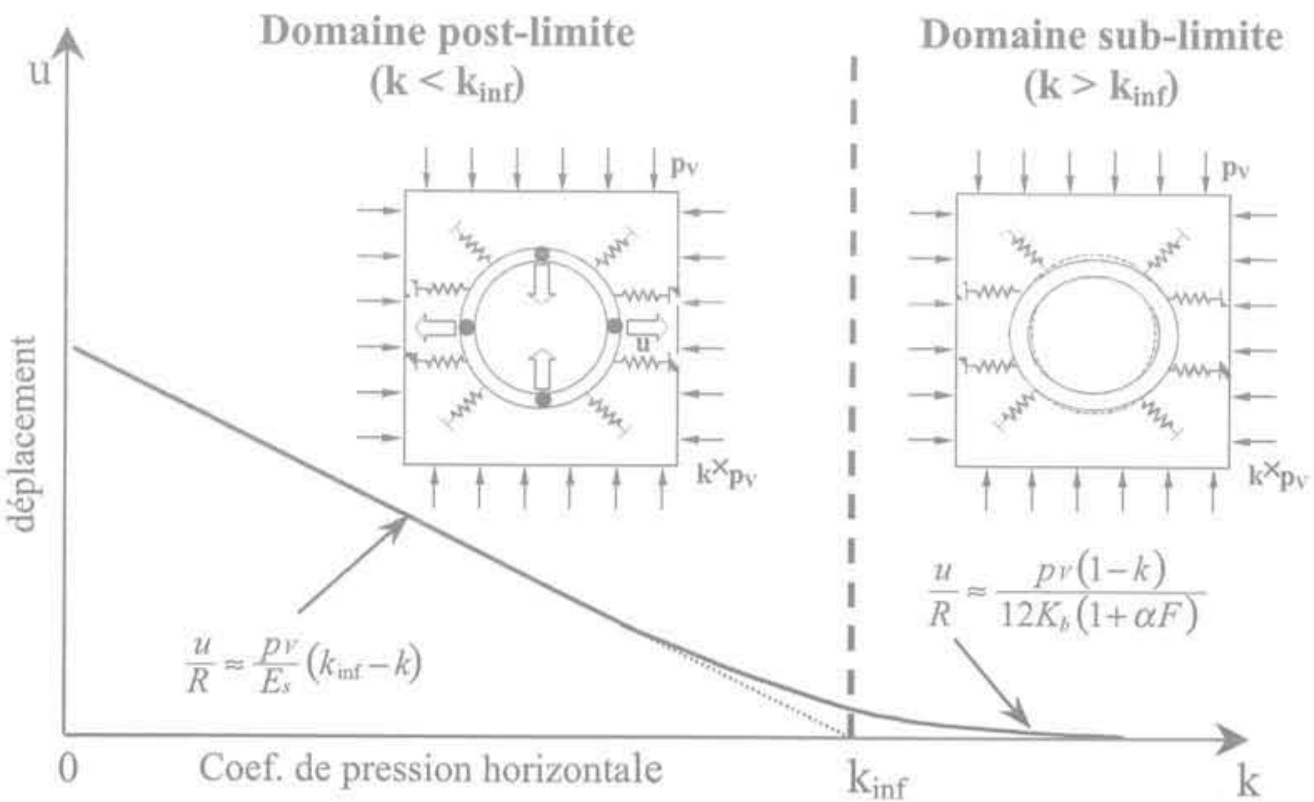

FG.9 Schématisation du fonctionnement mécanique de l'anneau dans les domaines post-limite et sub-limite. Shematic mechanical model of a masonry ring in the post-limit and the sub-limit domains. 
courbes de moment aux reins ne présentent pas de tangente horizontale, mais s'infléchissent aussi vers une asymptote quasi horizontale. L'allure de ces courbes s'interprète aisément à partir du diagramme de l'équilibre limite d'une section (Fig. 2b). On doit avoir:

$$
-\frac{h}{2} \leq \frac{M}{N} \leq \frac{h}{2}
$$

Ce qui donne:

- en clé-radier $M=k p_{v}\left(R+\frac{h}{2}\right) \frac{h}{2}$

et aux reins $\mathrm{M}=\mathrm{p}_{\mathrm{v}}\left(\mathrm{R}+\frac{\mathrm{h}}{2}\right) \frac{\mathrm{h}}{2}$

ou avec les valeurs numériques de l'exemple:

-en clé : $M=10,0 \times \mathrm{k}(\mathrm{kN} . \mathrm{m})$ et aux reins, $M=-10,0 \mathrm{kN} . \mathrm{m}$.

Ces deux droites sont représentées (en pointillés) sur la figure 10.

Le moment plastique en clé pour $\mathrm{k}=\mathrm{k}_{\mathrm{inf}}$ est donné par:

$$
\mathrm{mC}_{\mathrm{p}}=\mathrm{k}_{\text {inf }} \mathrm{p}_{\mathrm{v}} \mathrm{R}\left(\mathrm{R}+\frac{\mathrm{h}}{2}\right) \frac{\mathrm{h}}{2}=\frac{\mathrm{hR}}{2}\left(1-\frac{3 \mathrm{~h}}{2 \mathrm{R}}\right) \mathrm{p}_{\mathrm{v}}
$$

$=6 \mathrm{kN} \cdot \mathrm{m}$

Dans le domaine sub-limite $\left(k>k_{\text {int }}\right)$, le moment tend vers sa valeur élastique $M_{e}$ :

$$
M_{e}=\frac{1-k}{4} \frac{p_{V} R^{2}}{1+\alpha F}
$$

ou avec les valeurs numériques de l'exemple:

-en clé : $M=20,0 \times(1-k)(k N . m)$ et aux reins, $M=-20,0$ $x(1-$ k) kN.m.

Ces deux droites sont aussi représentées (en pointillés) sur la figure 10.
Contrairement au moment fléchissant, l'effort normal est peu sensible aux effets élasto-plastiques et à l'interaction sol structure. L'effort normal aux reins est proche de la valeur théorique $\mathrm{pv}(\mathrm{R}+\mathrm{h} / 2)=100 \mathrm{kN}$ et la valeur en clé est pratiquement égale à $100 \times \mathrm{k}$ kN. Cette relative insensibilité est due d'une part à la nature de l'effort normal, qui résulte du bilan des forces extérieures, et d'autre part, au fait que la rigidité normale d'un anneau est beaucoup plus élevée que sa rigidité en flexion.

\section{5}

\section{Analyse des contraintes}

On ne s'intéresse évidemment qu'aux contraintes de compressions qui sont maximales dans les sections situées aux reins. La figure 11 montre l'évolution de la contrainte maximale aux reins en fonction de $k$ pour les cas de figures prédéfinis dans cette étude (on rappelle que la pression verticale est égale à $100 \mathrm{kPa}$ soit approximativement le poids d'une colonne de $5 \mathrm{~m}$ de sol). Dans le cas d'un matériau raide $\left(E_{m}=10000 \mathrm{MPa}\right)$ et d'un sol lầche $\left(\mathrm{E}_{\mathrm{s}}=5 \mathrm{MPa}\right)$ la contrainte maximale de compression aux reins atteint une valeur relativement élevée lorsque $k$ tend vers zéro (de l'ordre de $10 \mathrm{MPa}$ ), qui pourrait entraîner la ruine par écrasement du matériau.

Jusqu'à présent, on n'a pas pris en compte la résistance en compression nécessairement finie du matériau sauf au premier chapitre où on a montré que les coefficients de poussée limite $\left(\mathrm{k}_{\text {inf }}\right.$ et $\mathrm{k}_{\text {sup }}$ ) sont peu influencés par la résistance finie du matériau. Cependant, l'éventualité d'un dépassement de la résistance en compression est tout à fait plausible et s'observe dans les cas

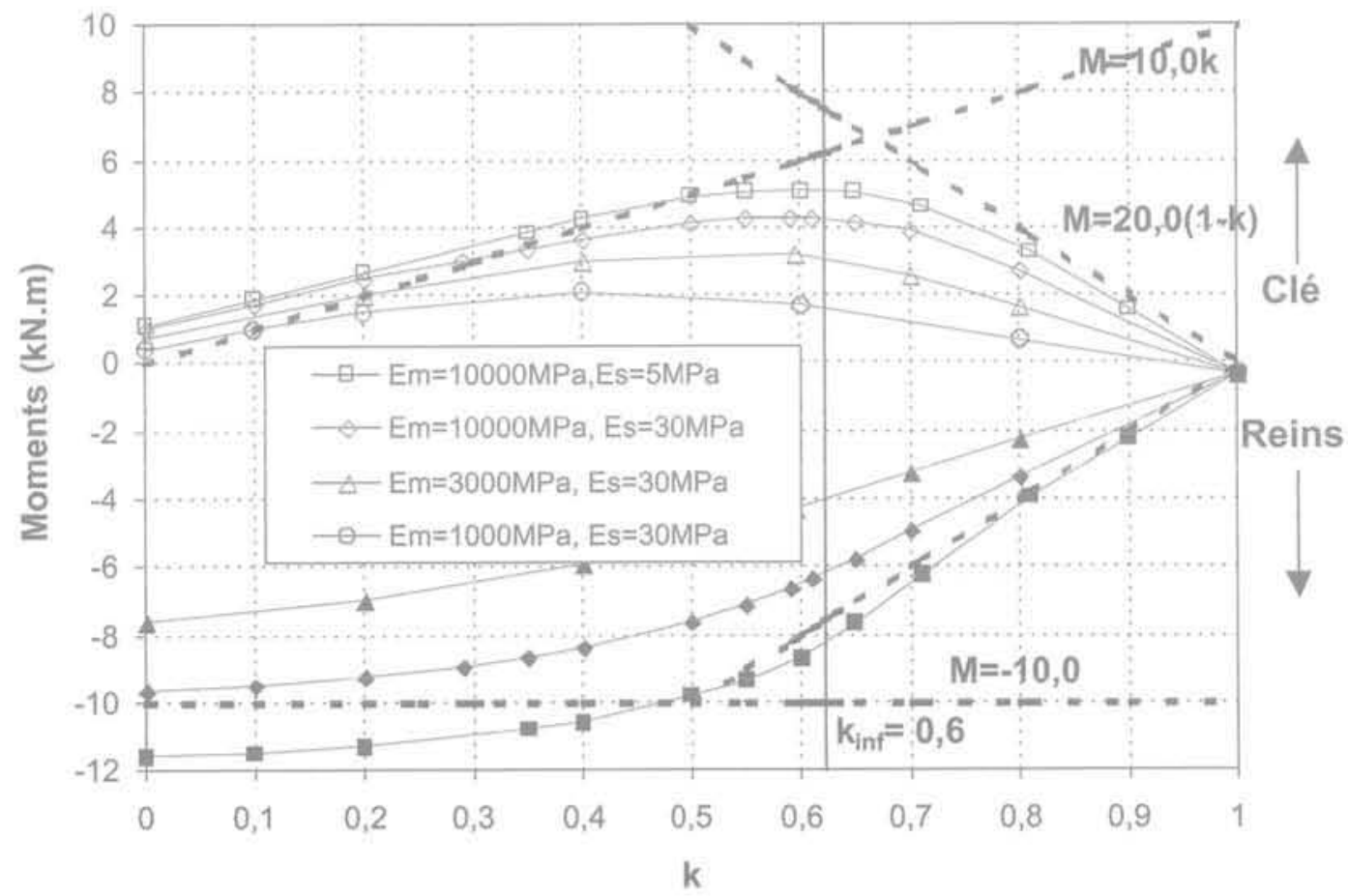

FiG, 10 Résultats des calculs par éléments finis : moments de flexion dans les sections de clè et de reins en fonction du coefficient de pression horizontale $\mathrm{k}$.

Finite element results: maximal bending moment at springlines and crown versus coefficient of horizontal earth pressure $k$. 


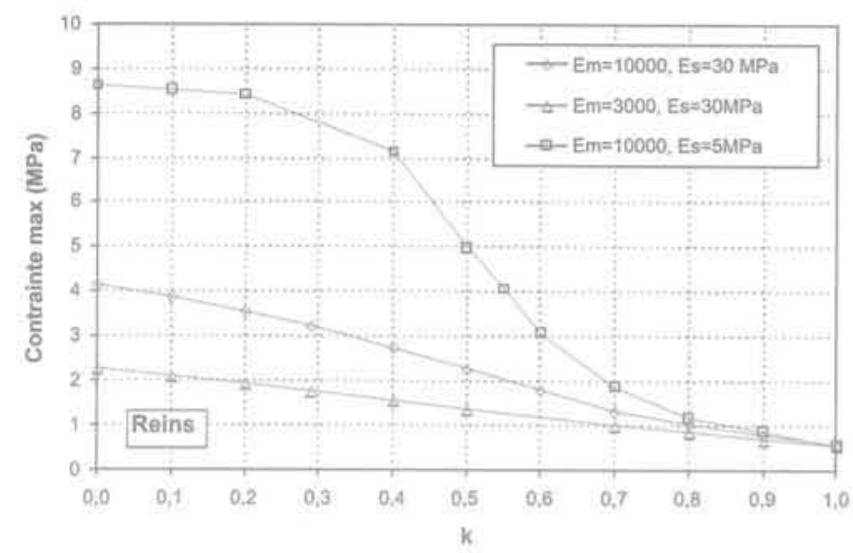

FiG. 11 Résultats des calculs par éléments finis : contrainte maximale de compression dans les sections de reins en fonction du coefficient de pression horizontale $\mathrm{k}$.

Finite element results: maximal compressive stress at springlines versus coefficient of horizontal earth pressure $\mathrm{k}$.

sévères d'ovalisation avec formation de fissures de (compression », dont le faciès est caractéristique (écaillage du parement notamment).

On présente dans Thépot (2004) un modèle analytique d'un système composé de quatre voussoirs articulés en interaction avec un massif élastique, qui permet de calculer la contrainte maximale de compression au niveau des sections les plus sollicitées selon l'expression suivante :

$$
\sigma_{c}(R)=\frac{p_{V} R}{h}\left[1+\sqrt{6(1-k) \frac{E_{m}}{E_{S}} \frac{h}{R}}\right]
$$

Cette formule montre que la contrainte maximale de compression doit varier avec la racine carrée du rapport entre le module de la maçonnerie $E_{m}$ et celui du sol $E_{s}$ ce qui est assez bien vérifié par les calculs aux éléments finis, dans le domaine post-limite $\left(\mathrm{k}<\mathrm{k}_{\mathrm{inp}}\right)$. En revanche, la formule prévoit des contraintes deux a trois fois supérieures aux résultats des calculs par éléments finis. En effet, dans le modèle analytique, la rotation des voussoirs est entièrement localisée au niveau des quatre sections fissurées (rotules) alors que dans le calcul aux éléments finis, avec une loi élasto-plastique parfaite, la rotation est distribuée sur toute la zone plastifiée qui s'étend progressivement à l'ensemble de l'anneau (seule une loi élasto-plastique à écrouissaqe négatif permettrait d'obtenir une localisation des déformations plastiques). Par conséquent, le calcul élasto-plastique sousestime la contrainte maximale de compression.

\section{Étude de la stabilité d'un ovoïde en maçonnerie par la méthode des éléments finis}

\section{Introduction}

On reprend l'analyse développée dans les deux chapitres précédents, mais appliquée à un ouvrage non circulaire en forme d'ovoïde, dont une coupe est représentée sur la figure 1 à droite, et qui est assez courant en région parisienne. La hauteur interne de l'ovoïde est de $2,3 \mathrm{~m}$, sa largeur aux naissances est de $1,3 \mathrm{~m}$ et l'épaisseur de la maçonnerie est de $20 \mathrm{~cm}$.

S'agissant d'un ouvrage non circulaire, on ne dispose pas de solutions analytiques explicites pour le calcul des sollicitations (moment, effort normal), la méthode des éléments finis est dans ce cas pratiquement indispensable.

\section{2}

\section{Caractéristiques du modèle aux éléments finis}

Les lois de comportement, les caractéristiques mécaniques et le chargement sont identiques à celles de l'étude de l'anneau circulaire, la figure 12 représente le maillage d'éléments finis. L'extrados de l'ovoïde est donc soumis à une distribution de pression et de cisaillement en équilibre avec un champ de contrainte dans le sol, qui se caractérise par des directions principales verticales et horizontales, une pression verticale $\mathrm{p}_{\mathrm{y}}$ égale à $0,1 \mathrm{MPa}$ et une pression horizontale égale à $\mathrm{k}$ fois la pression verticale. Comme dans l'étude précédente, on fait varier le coefficient $k$ et on maintient la pression verticale constante.

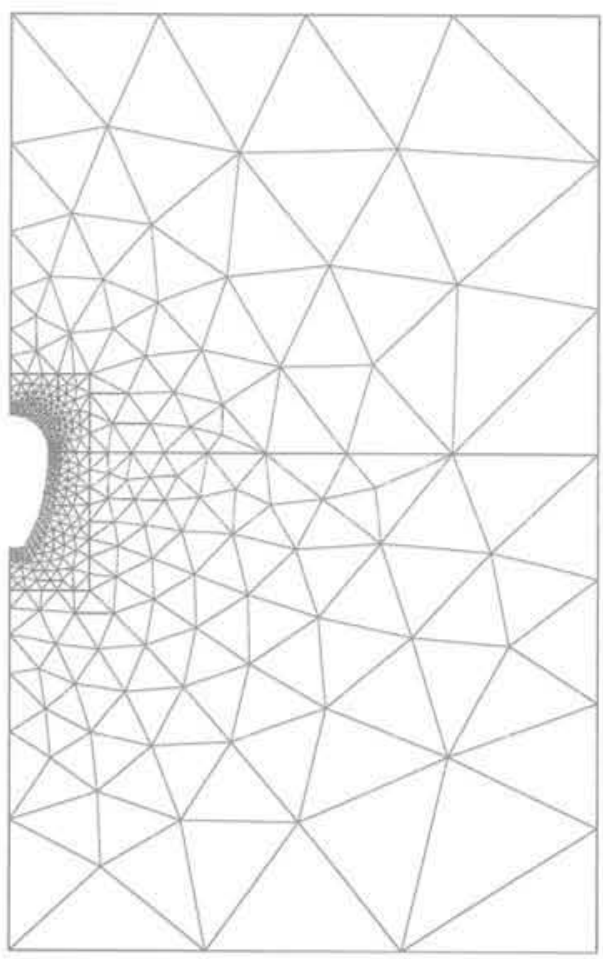

FIG. 12 Dessin du maillage d'éléments finis (logiciel CESAR-LCPC).

Drawing of the finite elements mesh (finite element package CESAR-LCPC). 


\section{Résultats des calculs: domaines sub-limite et post-limite}

\subsection{1.}

\section{Résultats sans interaction (module du sol égal à zéro)}

La figure 13 représente les déplacements horizontaux en fonction de k mesurés en deux points situés en milieu de piédroit et aux naissances de la voûte. On constate qu'il existe une borne inférieure égale à 0,25 et une borne supérieure égale à 0,5 pour lesquelles des déplacements divergent. La borne inférieure correspond à la ruine par affaissement de la voûte et divergence des piédroits, alors que la borne supérieure correspond à la ruine par convergence des piédroits (Figs, 14a et 14b),

Contrairement à l'anneau circulaire, où la borne supérieure est supérieure à 1 (et égale à l'inverse de la borne inférieure), les deux bornes sont ici inférieures à 1 et relativement proches l'une de l'autre. Le domaine de stabilité $\mathrm{n}$ 'inclut pas le cas $\mathrm{k}=1$ (pression uniforme) mais il est toutefois bien placé par rapport au coefficient de poussée au repos des terres qui varie typiquement de 0,3 à 0,5 . L'ovoïde est donc une forme doublement optimale à la fois du point de vue de l'action du sol et de son fonctionnement hydraulique.

\section{$4,3,2$}

\section{Résultats en prenant en compte l'interaction sol-structure}

Lorsque le module du sol est différent de zéro, on constate sur la figure 13 que les déplacements restent faibles dans la zone sub-limite et qu'ils augmentent rapidement quand k sort du domaine de stabilité naturelle. Dans les deux domaines post-limite (post-limite
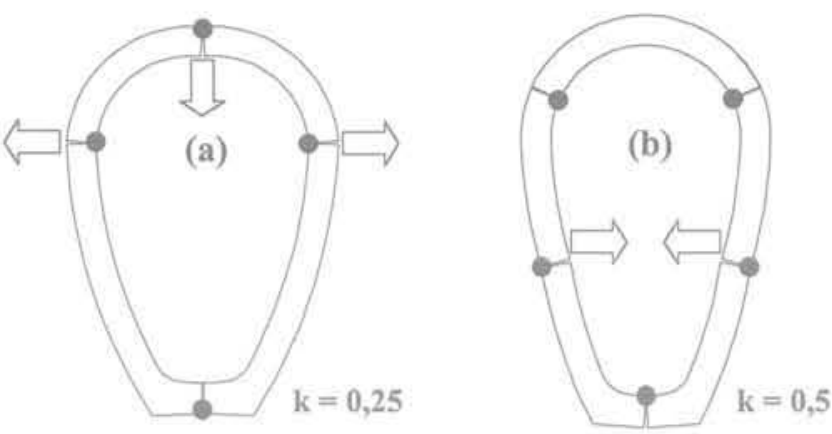

FG. 14 Configurations limites d'équilibre d'un ovoïde.

Ultimate limit states of an egg-shaped sewer.

inférieur pour $\mathrm{k}<0,25$ et post-limite supérieur pour $k>0,5)$, les déplacements varient quasi-linéairement avec $k$. On avait obtenu ce même résultat avec un anneau circulaire, et il se comprend bien, si on suppose que la formation de quatre rotules aux naissances-clé-radier pour $\mathrm{k}<0,25$ et en milieu de piédroits-naissances-radier, transforme l'ovoïde en un quasi mécanisme, dont la déformation est inversement proportionnelle au module du sol. Ce comportement est d'autant plus net que le module de la maçonnerie est élevé et celui du sol faible.

L'interaction sol-structure permet donc à l'ovoïde de trouver un équilibre en dehors de son domaine « naturel $»$ de stabilité. Finalement, on distingue troís domaines de fonctionnement (Fig. 15) : le domaine post-limite inférieur caractérisé par la divergence des piédroits et l'affaissement de la voûte, le domaine sublimite caractérisé par l'interaction quasi élastique avec le sol et enfin le domaine post-limite supérieur caractérisé par la convergence des piédroits. Les domaines post-limite sont aussi appelés domaines d'adaptation plastique de l'ovoïde.
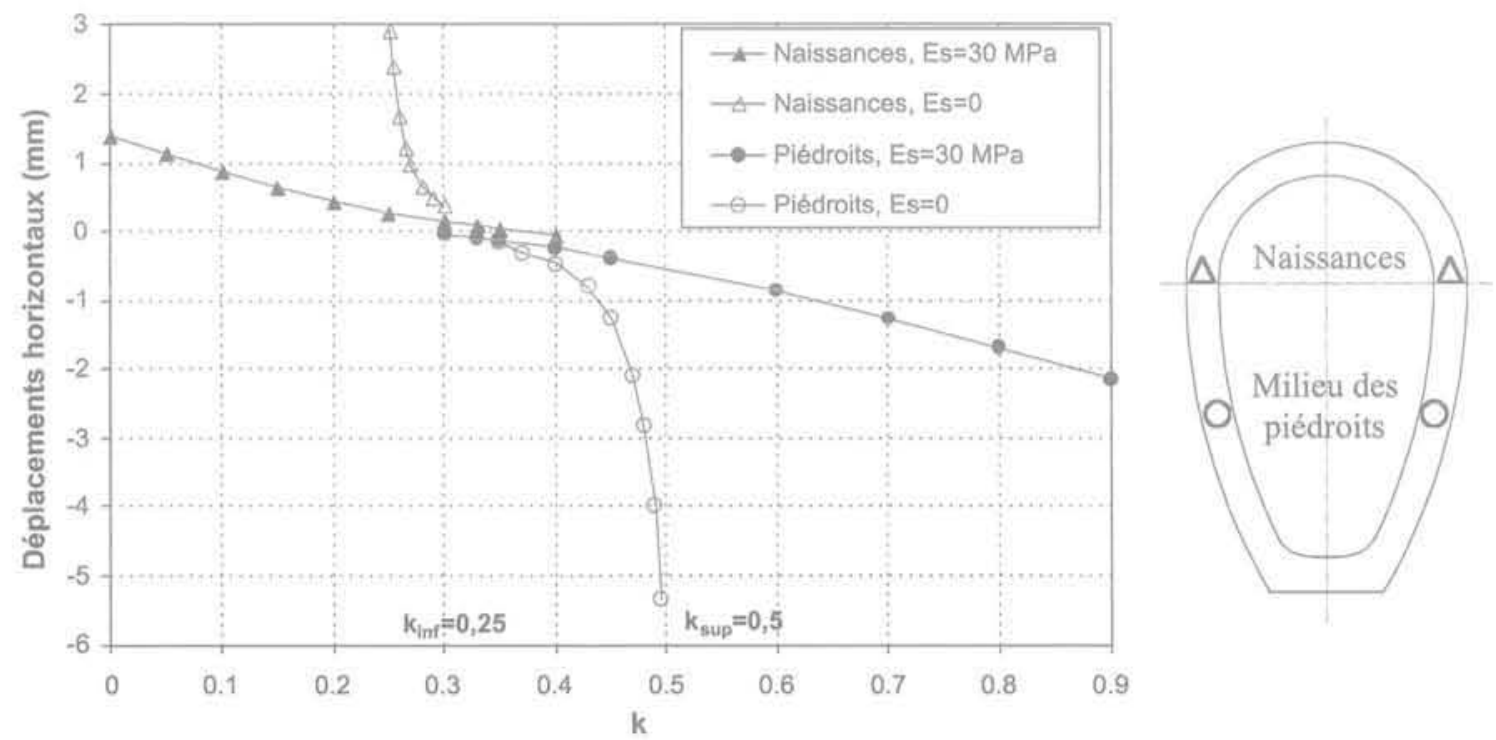

FIG. 13 Résultats des calculs par éléments finis avec interaction sol-structure : déplacements aux naissances et en milieu de piédroit.

Finite element results with soil-structure interaction: maximal deflection at springlines and haunch versus coefficient of horizontal earth pressure $k$. 


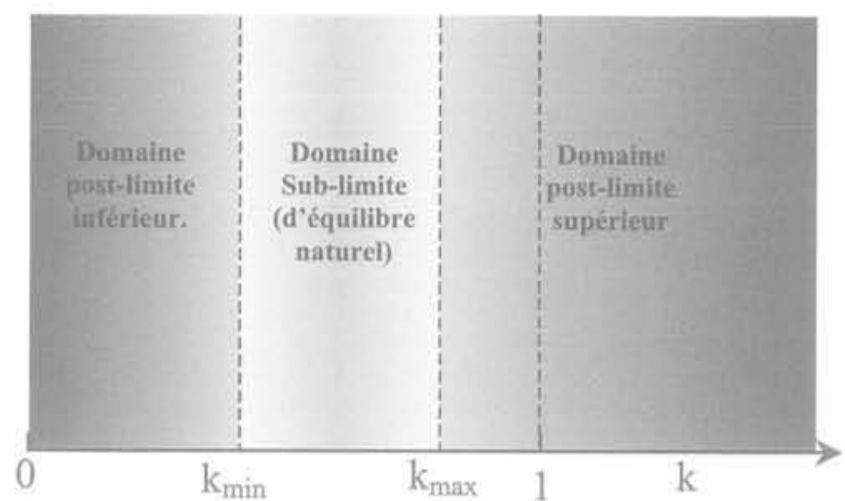

FIG. 15 Les trois domaines de comportement d'un ovoïde.

The three fields of behavior of an egg-shaped sewer.
Les valeurs habituelles de h/R relevées dans les ouvrages en maçonnerie sont en effet comprises entre 0,15 et $0,25(0,2$ est une valeur fréquente).

On constate donc que, pour les épaisseurs habituelles des conduites et collecteurs circulaires, l'équilibre d'un anneau en maçonnerie est généralement incompatible avec le coefficient de poussée des terres au repos. La portée de cet énoncé est évidemment affaiblie à plusieurs reprises par les hypothèses restrictives que l'on a prises sur le matériau maçonnerie (résistance à la traction nulle) et sur la géométrie du chargement. Mais il a l'avantage de montrer que dans de nombreux cas, l'équilibre d'un anneau en maçonnerie ne peut pas se concevoir sans prendre en compte l'interaction solstructure et la redistribution favorable des pressions qui en découle.

La méthode des éléments finis permet de prendre en compte l'interaction sol-structure et met clairement en évidence deux domaines de comportement : le domaine sub-limite, qui est aussi le domaine d'équilibre naturel de l'anneau borné par les valeurs $\mathrm{k}_{\text {inf }}$ et $\mathrm{k}_{\text {sun' }}$ où l'anneau a un comportement quasi élastique et le domaine post-limite où il tend à se comporter comme un ensemble de quatre voussoirs articulés en forte interaction avec le sol.

L'interaction avec le sol permet au ratio $k$ de passer les valeurs limites, mais on observe alors le développement rapide d'une plastification importante au niveau des sections situées aux quatre pôles de l'anneau (cléradier et reins) qui se transforme alors en un système de quatre voussoirs articulés avec quatre rotules dont la déformation est inversement proportionnelle au module du sol.

Dans le domaine sub-limite l'équilibre de l'anneau est donc totalement conditionné par la raideur du massif (l'écuilibre est d'ailleurs impossible si la raideur du sol est nulle). Le passage entre les deux domaines est d'autant plus brutal que le matériau est raide et le sol lâche.

On a représenté sur la figure 16 deux chemins possibles de chargement d'un anneau en maçonnerie dans le diagramme (h/R, k). Sur le premier chemin, l'état initial de l'anneau, représenté par le point (1a), est dans le domaine d'équilibre et on suppose que le ratio $k$ dimi-

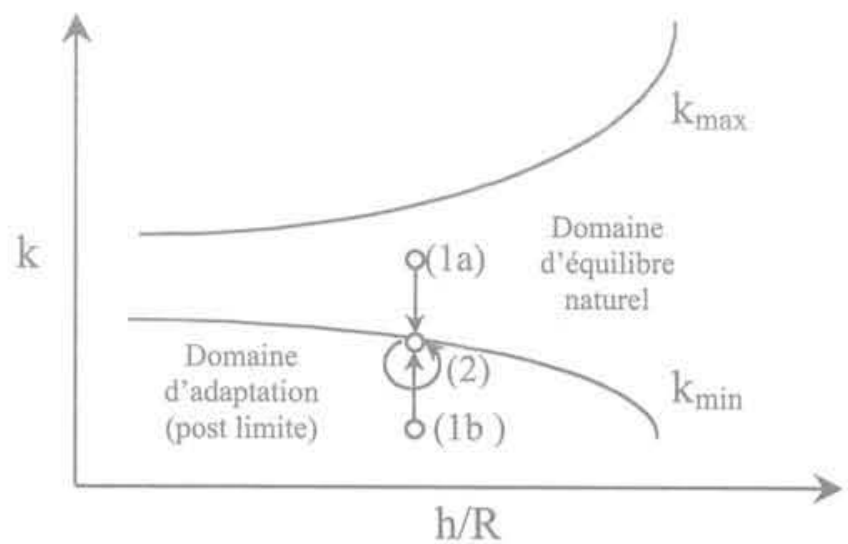

$$
\left(\frac{h}{R}\right)_{\min }=2\left(\frac{1-K_{0}}{3+K_{0}}\right) \text {. }
$$

Pour $\mathrm{K}_{0}=0,43\left(\varphi^{\prime}=35^{\circ}\right)$, valeur courante, on obtient un ratio épaisseur sur rayon moyen égal à 0,33 qui est une valeur très élevée, rarement observée en pratique.

$$
\mathrm{h}_{\min }=2 \mathrm{H}\left(\frac{1-\mathrm{k}}{3+\mathrm{k}}\right)
$$

Dans les méthodes de calcul des tunnels (AFTES, 1983) ou des conduites enterrées, on confond fréquemment le ratio $\mathrm{k}$ avec le coefficient de poussée des terres au repos $\mathrm{K}_{0}$ qui est classiquement donné par la formule de Jacky, pour un sol normalement consolidé: $K_{0}=\left(1-\sin \varphi^{\prime}\right)$ où $\varphi^{\prime}$ est l'angle de frottement.

$\mathrm{Si}$ on égale le $\mathrm{K}_{0}$ avec le $\mathrm{k}_{\text {inf }}$ qui correspond à l'équilibre limite de l'anneau en poussée minimum, on obtient la valeur minimum du ratio épaisseur sur rayon compatible avec un état de contrainte géostatique: 
nue, ce qui est représenté par la flèche verticale qui rencontre la ligne de poussée minimum en (2), où la structure est en état potentiel de rupture. La boucle symbolise le comportement post-rupture qui est généralement stable, car la déformation de la structure induit une redistribution du chargement qui maintient $k$ sur la ligne de poussée minimale. Sur le deuxième chemin, l'état initial de l'anneau représenté par le point (1b) est dans le domaine d'instabilité certaine, et on suppose que le ratio $k$ augmente jusqu'à ce qu'il atteigne la valeur minimale en (2).

Physiquement, le premier chemin peut par exemple se produire si on rajoute un remblai ou une surcharge sur un collecteur enterré dont le remblai latéral est compressible ou si on décompresse brutalement le remblai latéral (en ouvrant une tranchée parallèle par exemple). On obtient alors un "effet Marston» c'est-à- dire une concentration d'efforts sur la clé de la conduite et donc une diminution du ratio $k$. Le deuxième chemin peut se produire, au moment du décintrage de l'anneau, si le coefficient de poussée horizontale $\mathrm{K}_{0}$ des terres est inférieur au ratio de poussée minimum.

Finalement, cette étude montre que la stabilité d'un collecteur en maçonnerie (circulaire ou non circulaire) est très directement liée à la raideur du sol encaissant et qu'il n'y a généralement pas d'équilibre possible sans prise en compte de l'interaction sol structure:

Pratiquement, l'évaluation de la stabilité de ces ouvrages passe donc obligatoirement par des essais in situ visant à caractériser la déformabilité du sol encaissant (essai pressiométrique par exemple). Bien entendu, l'analyse de la fissuration et les mesures des épaisseurs et des qualités des maçonneries restent la base de toute évaluation.

\section{$\overline{\text { Bibliographie }}$}

AFTES - Recommandations sur l'emploi de la méthode convergence-confinement. Tunnels et ouvrages souterrains 59, 1983.

AFTES - Recommandations sur l'utilisa tion du béton non armé Tunnels et ouvrages souterrains 149, 1998.

Delbecq J.M. - Analyse dé la stabilité des voûtes en maçonnerie par le calcul à la rupture. Journal de mécanique théorique et appliquée 1, vol. 1, 1982, p. 91-121.

Einstein H.H. Schwartz C.W. - Simplified analysis for tunnel supports. Journal of Geotechnical Engineering Division, ASCE, vol. 105. GT4, 1979, p. 499-518.

Hervieux J. - Traité pratique de la construction des égouts. Ch. Béranger éditeur, 1897.

Heyman J. - The Masonry. Arch. Ellis Hor- wood Series in Engineering Science, 1982.

Humbert P. - CESAR-LCPC, un code général de calcul par éléments finis. Bulletin de liaison des LPC 160, 1989, p. 112 116.

Mestat P. - Lois de comportement des géomatériaux et modélisation par la méthode des éléments finis. Laboratoire central des ponts et chaussées, ERLPC, sèrie géotechnique, GT 52, 1993, 194 p.

Mestat P. - Etat de contraintes initiales dans les sols et calcul par èléments finis. Bulletin de liaison des LPC 215, mai-juin 1998 p. $15-32$

Panet M- Le calcul des tumnels par la méthode convergence-confinement. Presses des Ponts et Chaussées. 1995.

REPAU - Restructuration des collecteurs visitables, Guide technique, tome 2. Édition Tec et Doc, 2004.

Salençon J. - Calcul à la rupture et analyse limite. Presses des Ponts et Chaussées, 1983.

Save M. - La théorie des charges limites et son application aux maçonneries. Restauration des ouvrages et des structures, Presses des Ponts et Chaussées, 1983 p. 249-280.

Szechy K. - Traité de construction des tunnels. Dunod, 1970.

Thépot O. - Prise en compte des caractéristiques en petites déformations des sols dans l'étude du comportement des collecteurs enterrés. Thèse de doctorat, Ecole nationale des ponts et chaussées. 2004, p. 27-93. 